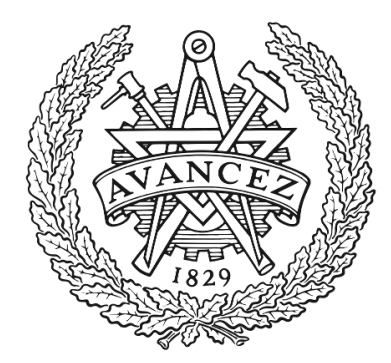

\title{
CHALMERS
}

UNIVERSITY OF TECHNOLOGY

\section{Spaceborne miniaturized UHF dual band helix antenna with a small frequency ratio}

Downloaded from: https://research.chalmers.se, 2023-04-26 12:19 UTC

Citation for the original published paper (version of record):

Xue, K., Liao, S., Zhu, R. et al (2021). Spaceborne miniaturized UHF dual band helix antenna with a small frequency ratio. Microwave and Optical Technology Letters, 63(6): 1767-1773.

http://dx.doi.org/10.1002/mop.32820

N.B. When citing this work, cite the original published paper. 


\section{Spaceborne miniaturized UHF dual band helix antenna with a small frequency ratio}

\section{Kai Xue ${ }^{1,2}$ (1) I Shaowei Liao ${ }^{3}$ () । Ruilong $\mathbf{Z h u}^{4}$ (1) । Quan $\mathrm{Xue}^{3}$ () । Liqin Ding $^{5}$ ( ) । Yang Wang ${ }^{1}$ ( ) । Qing Guo ${ }^{1}$ (ㅇ)}

\footnotetext{
${ }^{1}$ School of Electronics and Information Engineering, Harbin Institute of Technology Shenzhen, HIT Campus of University Town of Shenzhen, Shenzhen, Guangdong, China

${ }^{2}$ Shenzhen Aerospace Dongfanghong Satellite Ltd, Shenzhen, Guangdong, China

${ }^{3}$ Guangdong Provincial Key Laboratory of Millimeter-Wave and Terahertz, School of Electronic and Information Engineering, South China University of Technology, Guangzhou, Guangdong, China

${ }^{4}$ Xi'an matrix Wireless Technology Co., Ltd, Xi' an, Shannxi, China

${ }^{5}$ Department of Electrical Engineering, Chalmers University of Theology, Gothenburg, Sweden
}

\section{Correspondence}

Yang Wang, School of Electronic and Information Engineering, Harbin Institute of Technology Shenzhen, HIT Campus of University Town of Shenzhen, Nanshan, Shenzhen, 518055, Guangdong, China.

Email: yangw@hit.edu.cn

\section{Funding information}

National Science Foundation of China, Grant/Award Number: 61871189; Natural Science Foundation of Guangdong Province, Grant/Award Number: 2019A1515011999; Marine Economy Development Project of Guangdong Province under, Grant/Award Numbers: GDNRC [2020] 014, GDNRC [2020] 026; Science and Technology Project of Shenzhen under, Grant/ Award Numbers: JCYJ20170815140215733, JCYJ20200109113424990

\begin{abstract}
This study proposes a novel miniaturized circularly polarized (CP) ultrahigh frequency (UHF) quadrifilar helix antenna for spaceborne applications. The dual-band operation is realized using four inverted-U shaped helical strips (IUSHSs) that are rotated and alternately arranged on the four faces of a hollow polyimide cuboid in a sequential rotation manner, which effectively reduces the size of the antenna. Furthermore, the four IUSHSs are connected by a cross-shape strip at the top of the antenna to control the dual resonant frequencies, resulting in a small dual-band frequency ratio. The proposed antenna is both lightweight
\end{abstract}

and robust when compared with the conventional miniaturized CP antennas operating at similar bands with similar performance. In particular, its compact radiator provides effective miniaturized spaceborne solution without the need of high-dielectric coefficient materials. A device for spaceborne application that operates at $402 / 505 \mathrm{MHz}$ is designed, fabricated, measured, and inorbit tested with a weight of $651 \mathrm{~g}$ and an effective size of $0.161 \times 0.161 \times 0.228 \lambda^{3}{ }_{402 \mathrm{MHz}}\left(\lambda_{402 \mathrm{MHz}}\right.$ is the wavelength at $402 \mathrm{MHz}$ ). The measured gain and axial ratio of the proposed antenna are better than $5.32 \mathrm{dBi}$ and $2.18 \mathrm{~dB}$, respectively, within 2 and $12 \mathrm{MHz}$ bandwidth for the two bands. The test results proved that the methods used to design the proposed antenna are effective.

\section{KEYWORDS}

dual-band operation, quadrifilar helix antenna, small frequency ratio, spaceborne, UHF band

\section{1 | INTRODUCTION}

Ultrahigh frequency (UHF) bands cover a frequency range of $0.3-1 \mathrm{GHz}$ according to IEEE standards. Although inherently low in data rate, spaceborne UHF datalinks are expected to be operationally available for a long time, as the EM wave of the band can easily penetrate obstacles, thereby providing a stable wireless linkage. ${ }^{1,2}$

Quadrifilar helical antennas (QHAs) with circularly polarized $(\mathrm{CP})$ radiation are widely used in tracking telemetry and command (TT\&C), satellite navigation systems, and data collection systems (DCSs). Presently, with the DCS development of low earth orbit microsatellites, wide hemispherical beamwidths, and a small frequency ratio are generally required for spaceborne antenna applications. However, conventional miniaturized QHAs endure narrow bandwidths and ill-matched input impedances because of their operation at resonant modes instead of traveling modes. ${ }^{3,4}$

This study proposes an innovative miniaturized QHA for a small/micro satellite to satisfy the specifications: operating frequency of $401-403 \mathrm{MHz}, 499-511 \mathrm{MHz} ; 3 \mathrm{~dB}$-axial-ratio beamwidth of $-60^{\circ} \leq \theta \leq 60^{\circ}$ (LHCP); gain of $\geq 3 \mathrm{dBi}$ at broadside, $\geq-2.5 \mathrm{dBi}$ at $-60^{\circ} \leq \theta \leq 60^{\circ} ; \leq 0.142 \times 0.142 \times 0.211$ $\lambda^{3}{ }_{402 \mathrm{MHz}}\left(\lambda_{402 \mathrm{MHz}}\right.$ is the wavelength at $\left.402 \mathrm{MHz}\right)$, or $\leq 106 \times 106 \times 157 \mathrm{~mm}^{3}$ ). A compact electrical size of 0.142 
$\lambda_{402 \mathrm{MHz}}$, and a low dual-band frequency ratio of 1.25 attribute to the complex design.

Various miniaturized QHAs based on dielectric loading insertion, ${ }^{5}$ application of different helix turn angles, ${ }^{6}$ helix

(A)

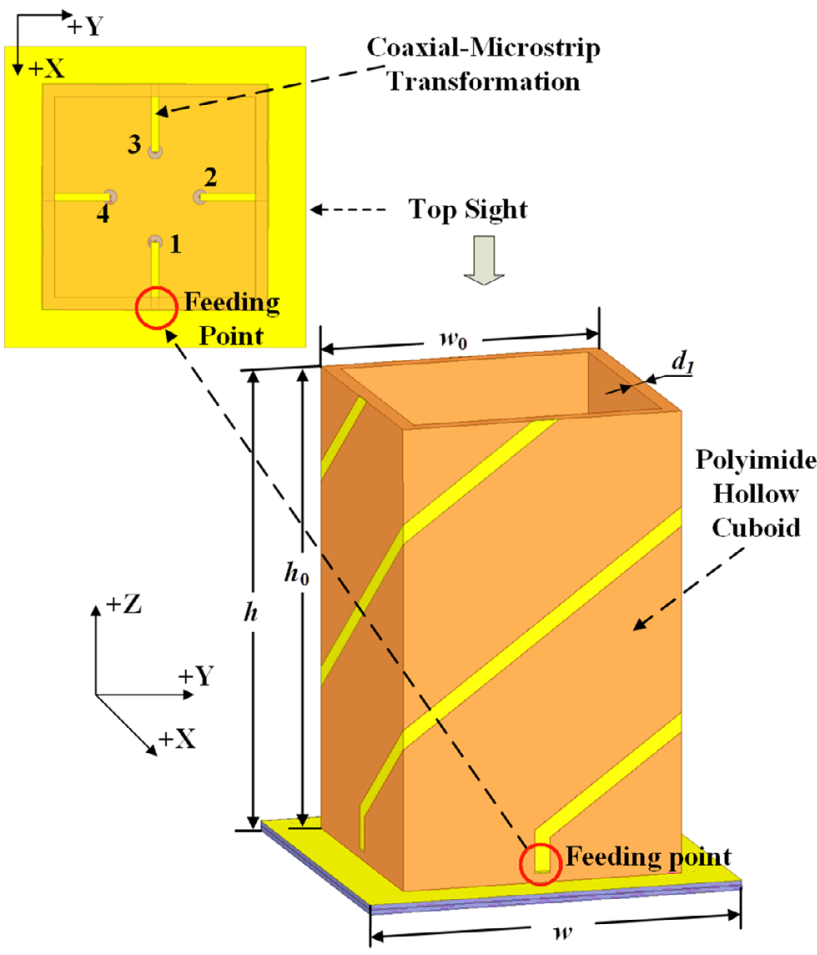

Step1: Four single-helical metal strips

(C)
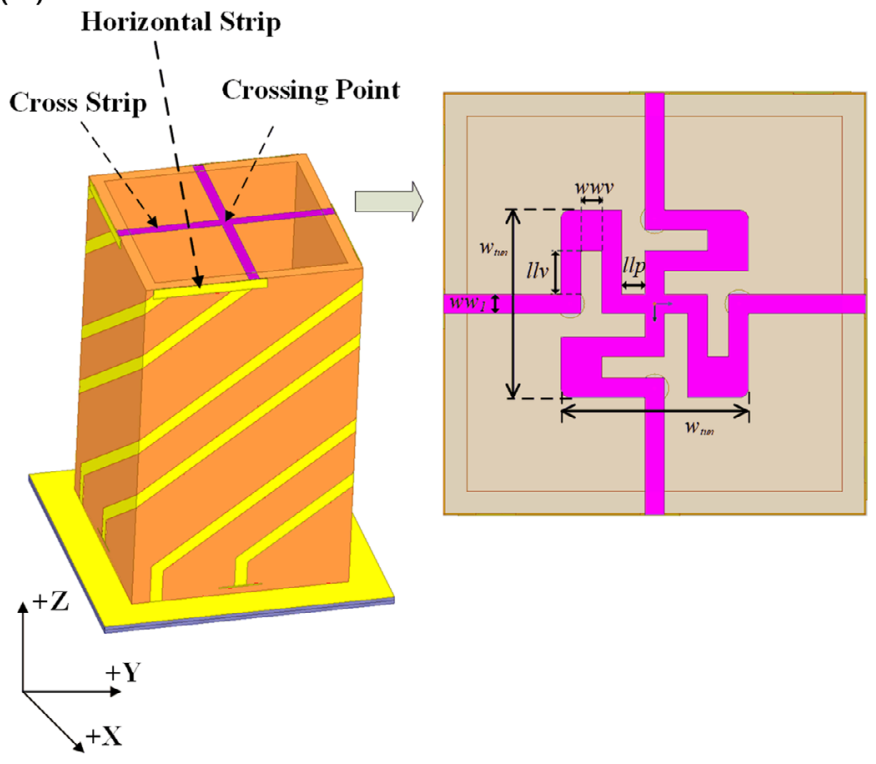

Step3: Adding top cross-shape strip antenna arms folding, ${ }^{7}$ inverted-F monopoles folding, ${ }^{8}$ and power divider networks ${ }^{9}$ have been proposed. However, miniaturization constricts the bandwidth. To overcome this drawback, addition of a parasitic helix arm to broaden the bandwidth, ${ }^{10-13}$
(B)

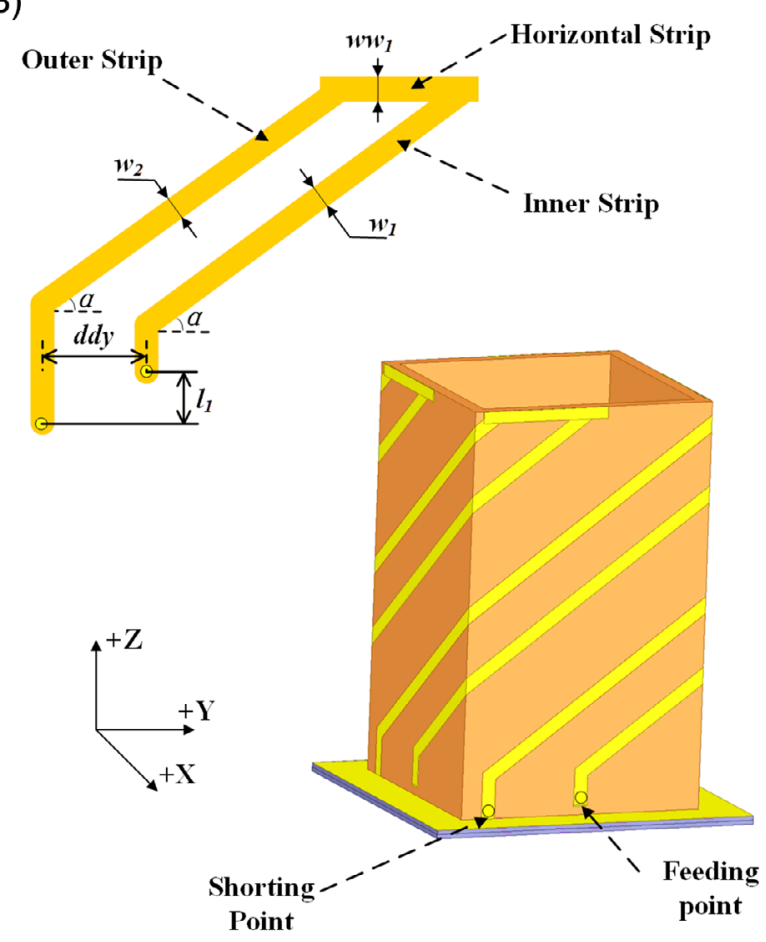

Step2: Four special-designed IUSHSs

(D)

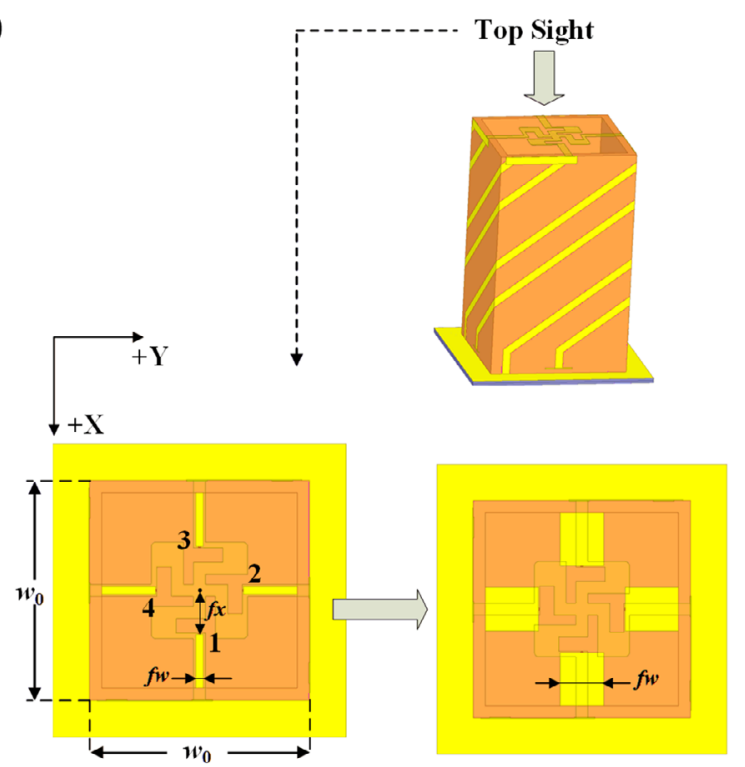

FIGURE 1 Evolution steps of proposed QHA: (A) Four single-helical metal strips; (B) Four special designed IUSHSs; (C) Adding top crossshape strip; and (D) Feeding port matching. ( $w_{0}=90 \mathrm{~mm}, h_{0}=149 \mathrm{~mm}, d_{1}=5 \mathrm{~mm}, \alpha=35^{\circ}, d d y=39 \mathrm{~mm}, l_{1}=10 \mathrm{~mm}, f w=19 \mathrm{~mm}$, $w_{1}=7 \mathrm{~mm}, w w_{1}=5 \mathrm{~mm}, w_{2}=5 \mathrm{~mm}, w_{\text {tun }}=39.5 \mathrm{~mm}$ ). IUSHS, inverted-U shaped helical strips; QHA, quadrifilar helical antennas [Color figure can be viewed at wileyonlinelibrary.com] 

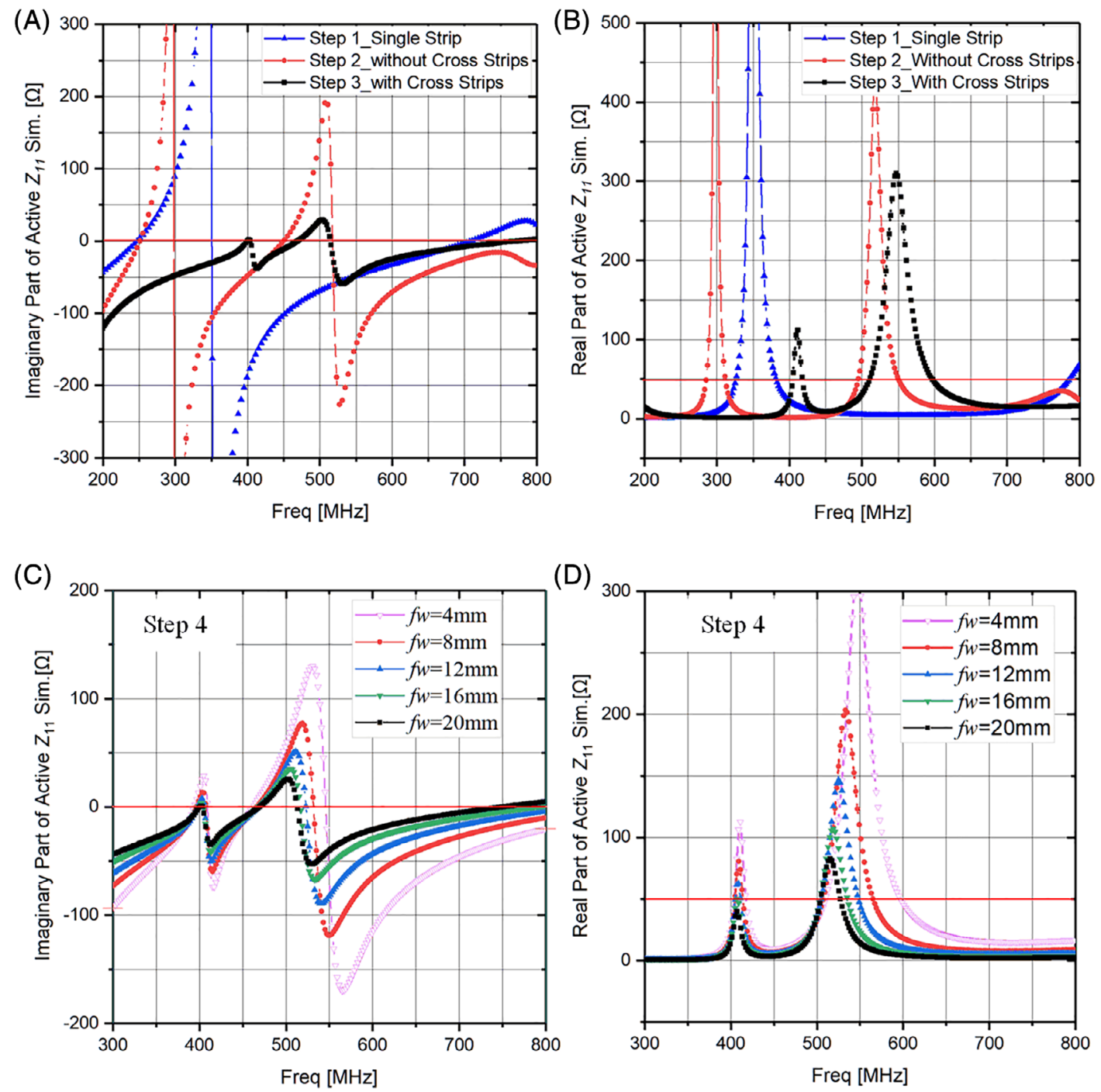

FIG URE 2 Simulated imaginary and real parts of Active $Z_{11}$ : (A) $\operatorname{Im}\left[\right.$ Active $Z_{11}$ ] of steps 1-3. (B) $\operatorname{Re}\left[\right.$ Active $\left.Z_{11}\right]$ of steps $1-3$. (C) $\operatorname{Im}[$ Active $Z_{11}$ ] under different $f w$ (step 4). (D) Re[Active $Z_{11}$ ] under different $f w$ (step 4) [Color figure can be viewed at wileyonlinelibrary.com]

use of stepped-width arms,${ }^{14}$ or use of a dual-faced slot radiation structure $^{15}$ to realize dual-band operation are proposed. Nevertheless, the performance cannot be satisfied using these designs. Therefore, this study focuses on implementing innovative designs. Specifically, it aims to design a spaceborne miniaturized UHF dual-band antenna to overcome existing challenges.

\section{2 | DESIGN OF THE ANTENNA}

QHA is a circular polarization (CP) antenna, which is widely used in spaceborne applications. It primarily consists of a four-element radiator and a feeding network. The radiator is generally constructed using helical conducting strips on a dielectric substrate. ${ }^{12,13}$ The feeding network provides a multiway signal with an equal amplitude and a progressive phase shift to drive the radiator. Miniaturized QHA is a resonant antenna. Therefore, its dimensions must be selected appropriately to obtain optimum characteristics in its operating frequency band.

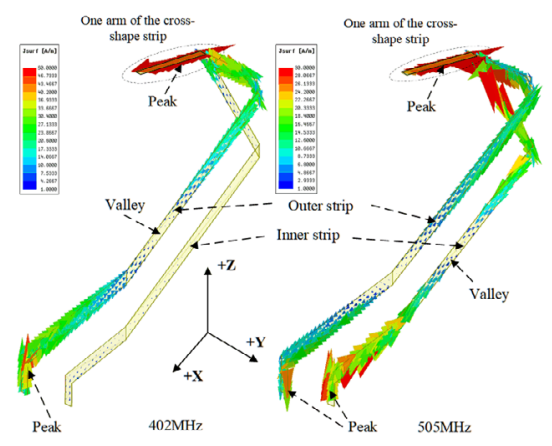

FIGURE 3 Resonance mode on the combination of cross-shape strip and IUSHS at 402/505 MHz (current distribution). IUSHS, inverted-U shaped helical strips [Color figure can be viewed at wileyonlinelibrary.com]

\section{1 | Radiator design and experiments}

In this section, a miniaturized radiator is presented and discussed, which utilizes four inverted-U shaped helical strips (IUSHSs) to reduce the size and operate at the dual band as 
well as a cross-shaped strip between the four IUSHSs to reduce the resonant frequency ratio. The evolution of the design is described below.

The structural dimension requirement of the proposed QHA is shown in Figure 1(A). The radiator is composed of four IUSHSs, which are wounded as four helixes on a supporting hollow cuboid polyimide frame with limited side length $w \leq 106 \mathrm{~mm}$, height $h \leq 157 \mathrm{~mm}$. For an electrically small antenna, the larger the size, the better the performance. The proposed QHA is designed according to the maximum acceptable size, $w=106 \mathrm{~mm}, h=157 \mathrm{~mm}$, and frame thickness $d_{1}=5 \mathrm{~mm}$.

Initially (step 1), a traditional miniaturized QHA radiator was designed, as shown in Figure 1(A). It forms four straight helical strips, where each strip has approximately $\lambda / 4$ and 1/2 turn, with an opening at the top. The feeding point is arranged at the center of the bottom edge of the cuboid flank. The resonance point is defined as the frequency at which the imaginary part of the input impedance $\left(\operatorname{Im}\left[\right.\right.$ Active $\left.\left.Z_{11}\right]\right)$ is zero and the real part $\left(\operatorname{Re}\left[\right.\right.$ Active $\left.\left.Z_{11}\right]\right)$ is none zero. Simulated $\operatorname{Im}$ [Active $Z_{11}$ ] is shown in Figure 2(A). A resonant point is observed at 240 and $700 \mathrm{MHz}$ with a frequency ratio of 2.91. Furthermore, Re [Active $Z_{11}$ ] is much larger than $50 \Omega$, and $\operatorname{Im}$ [Active $Z_{11}$ ] changes rapidly with frequency (Figure 2(B)), resulting in a poor match at resonant points.

Subsequently (step 2), to realize dual-band operation, an unconventional helical conducting strip, IUSHS, is designed. The expanded view of an IUSHS is shown in Figure 1(B). Each IUSHS with inclination angle $\alpha$, is fed with a coaxial port at one end, followed by an elongation and $180^{\circ}$ rotation at the other end; it is then shorted to the ground near the feeding point. The elongated and original parts are parallel. Briefly, each rotated metal strip consists of an inner strip, an outer strip, and a horizontal strip with widths $w_{1}, w_{2}$, and $w w_{1}$, respectively. The horizontal and vertical distances between the feeding and shorting points are denoted as $d d y$ and $l_{1}$. This introduces a resonance point of 298 and $515 \mathrm{MHz}$, with a frequency ratio of 1.73 (Figure 2(A)). However, it still does not satisfy the requirement, and $\operatorname{Re}\left[\right.$ Active $Z_{11}$ ] is far from $50 \Omega$ (Figure 2(B)), resulting in a poor match.

To effectively reduce the frequency ratio, a crossshaped strip is connected to the horizontal strip of the separated IUSHSs at the top (step 3), as shown in Figure 1(C). The center of the cross-shaped strip is equivalently shorted to the ground, as the phase difference between each two separated IUSHSs is $180^{\circ}$. From Figure 3, the simulated current distributions on an IUSHS shows two different resonant energy distributions at 402 and $505 \mathrm{MHz}$ : one is primarily at the outer strip, while the other is at both strips. Consequently, the resonant points can be tuned independently, to compensate frequency deviation caused by fabrication errors. The current density distribution on the combination of crossshape strip and IUSHS operates in dual $\lambda / 4$ resonant mode, as shown in Figure 3. Hence, the dimensions of the cross-shape strip will affect the resonant frequency of the radiator. Correspondingly, the geometry dimension of the cross-shape strip is as shown in Figure 1(C), where $w w v=w_{\text {tun }} / 2-w w_{1} / 2-l l p-2 * w w_{1}, l l v=l l p+w w_{1}$, $l l p=w_{\text {tun }} / 10$. The impact of the parameters $w_{\text {tun }}$ and $w w_{1}$ on the resonant frequencies have been studied. The dual resonant frequencies will increase simultaneously with the increase of $w_{\text {tun }}$. While, $w w_{1}$ has monotonous impact to the lower resonant frequency without affecting the higher one. Therefore, independent tuning of two frequencies can be realized by appropriately adjusting the
(A)

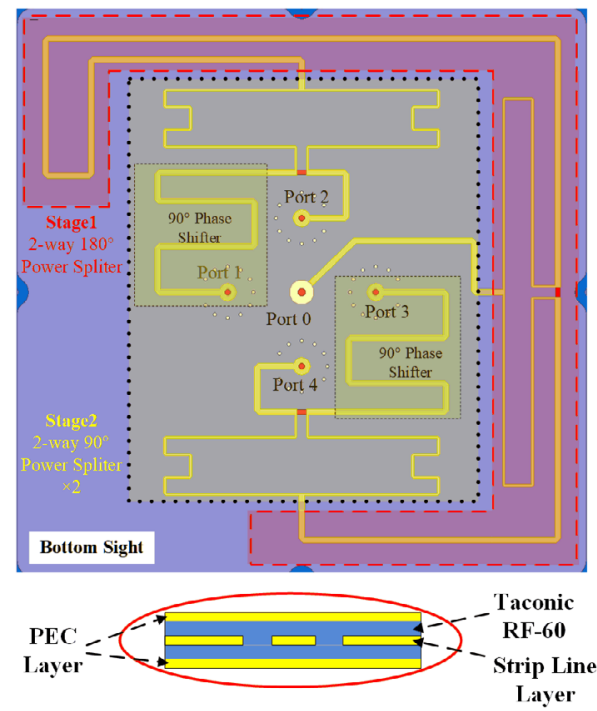

(B)

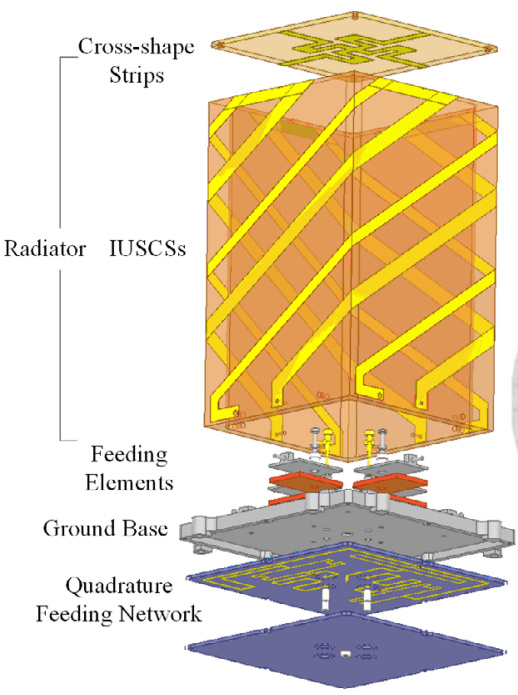

(C)

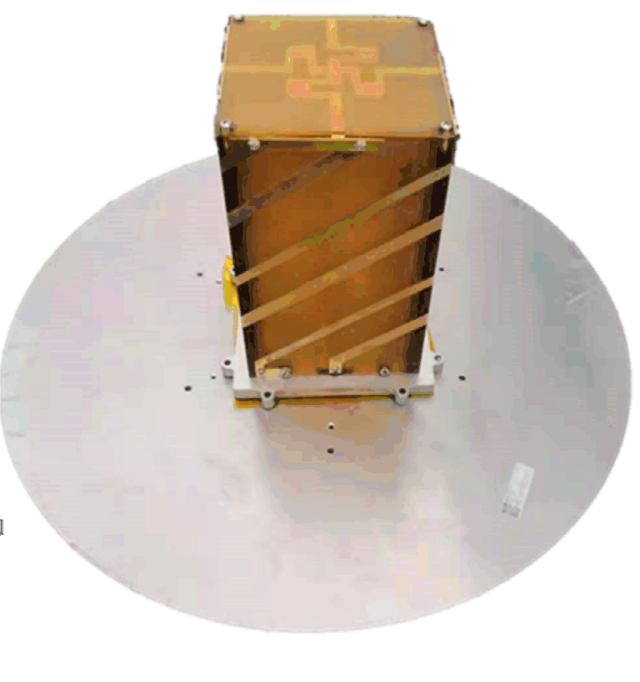

FIGURE 4 Fabricated prototype. (A) Explosive view of the model. (B) The feeding network. (C) Photograph of the fabricated prototype mounted on a $\varphi=350 \mathrm{~mm}$ metal ground. IUSHS, inverted-U shaped helical strips [Color figure can be viewed at wileyonlinelibrary.com] 

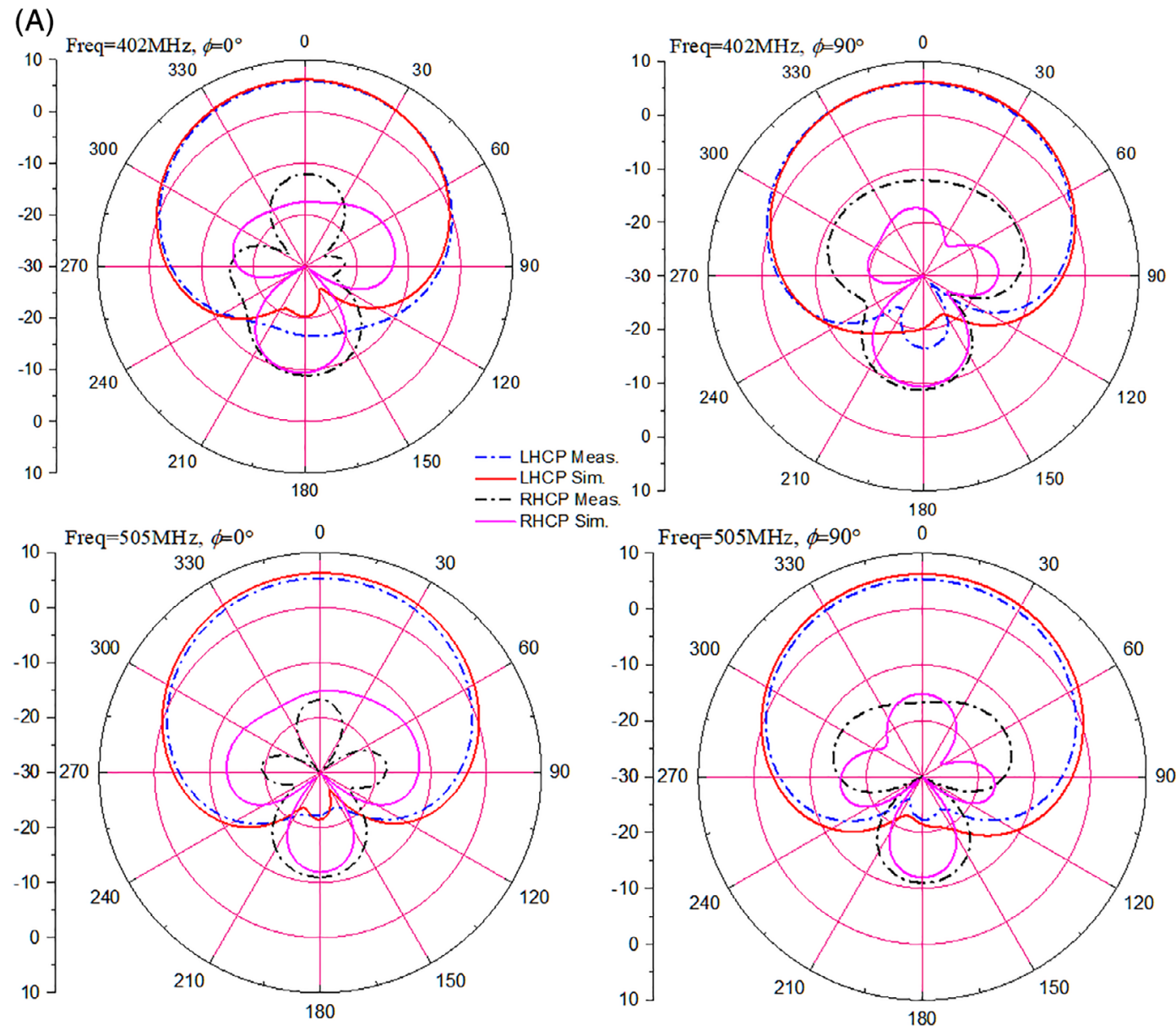

(B)

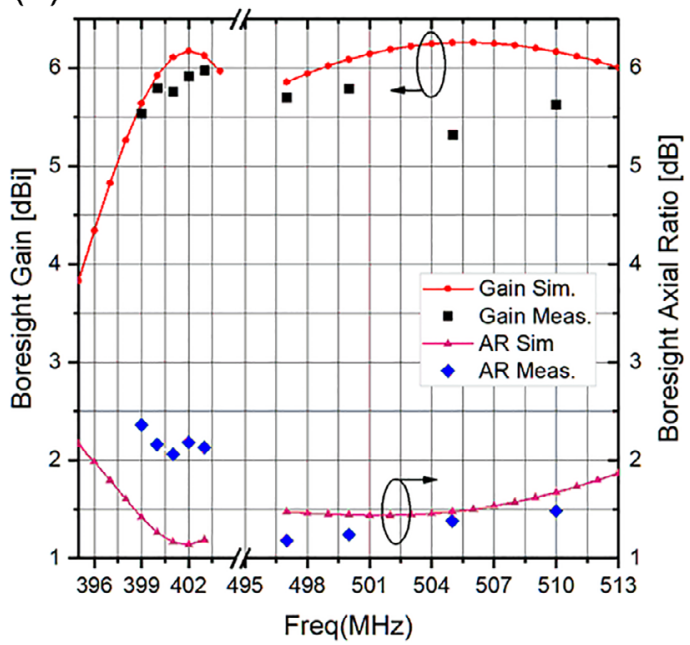

(C)

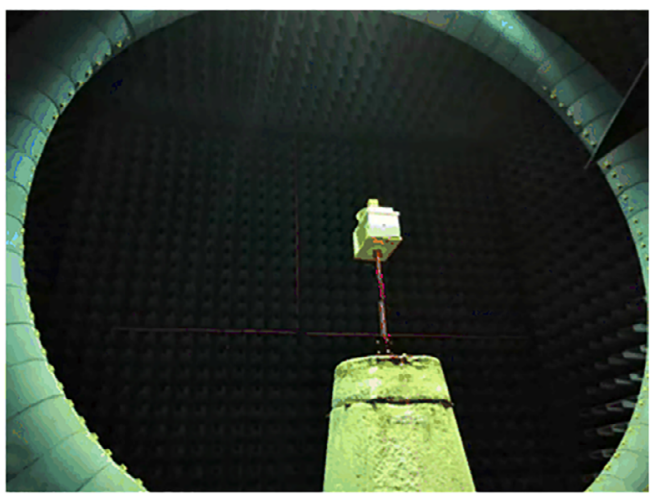

F IGURE 5 Radiation characteristics (sim. vs. meas.) and measurement environment. (A) Radiation patterns on $\varphi=0^{\circ} / 90^{\circ}$ plane at $402 /$ $505 \mathrm{MHz}$. (B) Broadside gain and AR. (C) Photograph of the prototype under measurement. AR, axial ratio [Color figure can be viewed at wileyonlinelibrary.com]

two parameters. Simultaneously, impedance matching is improved; however, the obtained impedance matching is still unsatisfactory (Figure 2(B)).

Finally (step 4), to improve the impedance matching of the radiator, the width of the transforming line between the feeding point of the element and the coaxial feeding point is optimized as shown in Figure 1(D). It can be seen from Figure 2(C),(D) that $\operatorname{Re}\left[\right.$ Active $Z_{11}$ ] reaches $50 \Omega$ while Im [Active $Z_{11}$ ] slightly varies at 402 and $505 \mathrm{MHz}$, as $f w$ varies from 5 to $20 \mathrm{~mm}$. 
TABLE 1 Comparison of the proposed QHA and previously reported QHAs

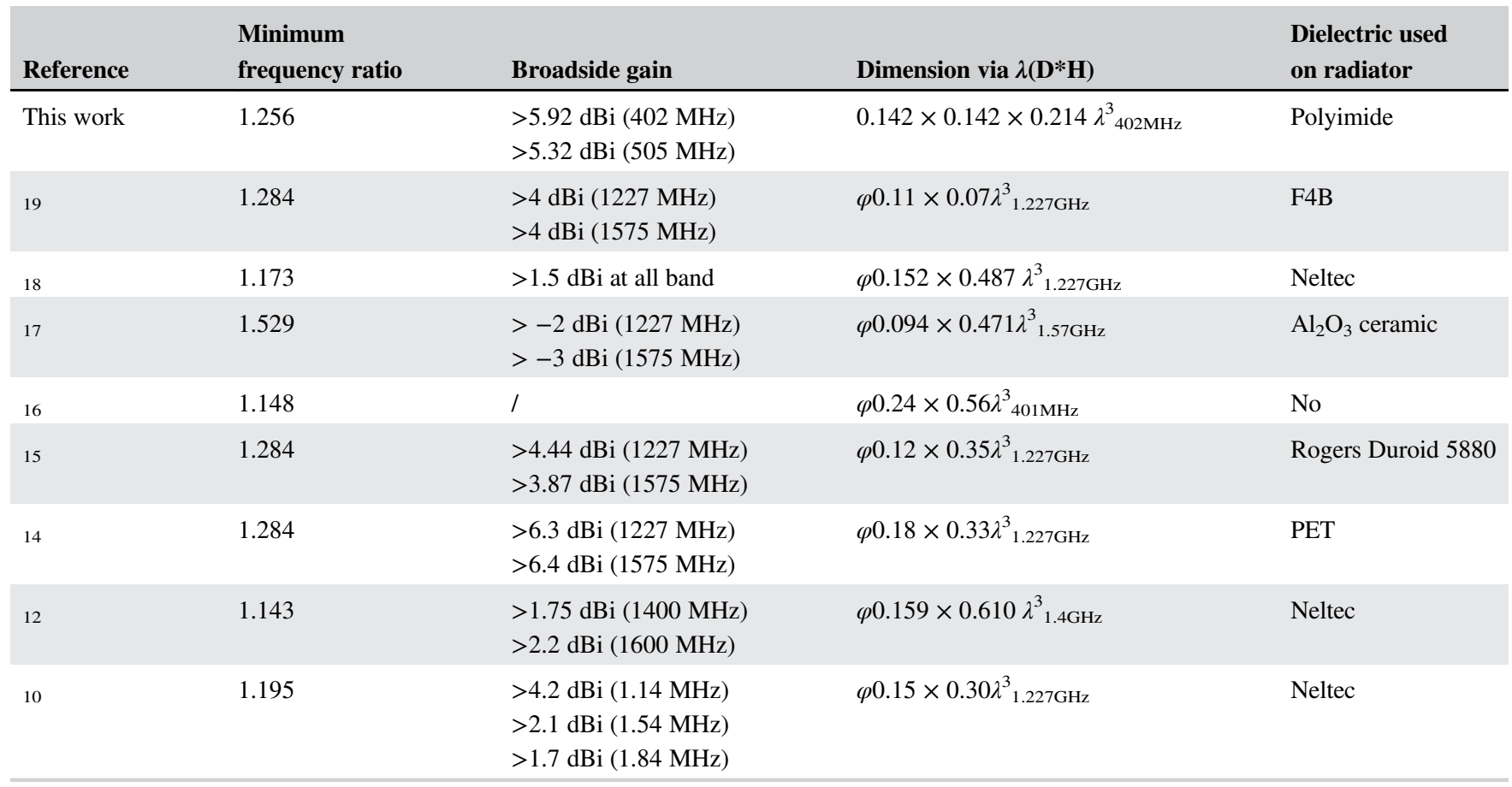

\section{2 | Antenna assembly and consolidation for spaceborne applications}

A quadrature feeding network is needed to provide equal amplitude signals with sequential phases of $0^{\circ}, 90^{\circ}, 180^{\circ}$, and $270^{\circ}$. As shown in Figure 4(A), it is implemented on two Taconic RF-60 substrates, which consists of two-stage Wilkinson power divider cascaded with phase shifters. Simulation shows that a low insertion loss of approximately $0.2 \mathrm{~dB}$ was obtained, and the maximum magnitude and phase imbalance are $0.1 \mathrm{~dB}$ and $33.6^{\circ}$ respectively. An SMA connector connecting Port 0 of the feeding network is fastened on its back.

The proposed antenna can be assembled by mounting the feeding network on the bottom of the radiator, as shown in Figure 4(B),(C), which has a dimension of $106 \times$ $106 \times 157 \mathrm{~mm}^{3}$ (excluding $\varphi=350 \mathrm{~mm}$ metal ground) or $0.142 \times 0.142 \times 0.211 \lambda^{3}{ }_{402 \mathrm{MHz}}$ and a weight of $786 \mathrm{~g}$.

To meet the mechanical, thermal, and radiational reliability requirements in spaceborne applications, an engineering design is realized. The fabricated radiator here is slightly different from that of Figure 1 in that all surface components are assembled by means of screw joints instead of welding, solder joints are fastened by screws before welding, immune-to-radiation polyimide is used to support IUSHSs.

\section{3 | Antenna measurements}

The prototype is placed in the center of a $\varphi=350 \mathrm{~mm}$ metal ground to emulate the satellite body when measured, as shown in Figure 4(C). The measured reflection coefficient is less than $-15 \mathrm{~dB}$ at $401-511 \mathrm{MHz}$, similar to the simulated result of the feeding network.
Subsequently, the measured radiation performance is shown in Figure 5. The measured normalized LHCP (main polarization) radiation patterns at the two bands are in agreement with the simulated ones. Measured broadside gains are $5.92 \mathrm{dBi}$ at $402 \mathrm{MHz}$ and $5.32 \mathrm{dBi}$ at $505 \mathrm{MHz}$, respectively. It can be observed that they are approximately 0.2 and $0.9 \mathrm{~dB}$ lower than the simulated ones. The measured broadside axial ratios (ARs) are higher than 2.18 and $1.38 \mathrm{~dB}$ at 402 and $505 \mathrm{MHz}$, while simulated ones are 1.14 and $1.48 \mathrm{~dB}$. Within 2 and $12 \mathrm{MHz}$ bandwidths at the two bands, measured broadside gains are larger than 5.76 and $5.32 \mathrm{dBi}$ respectively, which are 0.34 and $0.7 \mathrm{~dB}$ lower than the simulated ones. For $\mathrm{AR}$, the measured results are 2.18 and $1.48 \mathrm{~dB}$, while simulated ones are 1.19 and $1.74 \mathrm{~dB}$. Measured radiation characteristics are slightly deviated from simulated ones because of the errors from dielectric coefficient, fabrication, and assembling. However, these errors also cause that measured AR is better than simulated one because of the better amplitude and phase imbalance of the fabricated prototype than those of the simulation model. Thus, it can be confirmed that the prototype can well satisfy the requirements presented in the introduction.

Space environment tests on sinusoidal vibration, random vibration, and thermal vacuum cycling were conducted for the fabricated prototype. The measured results show negligible differences between the performances of the antenna system before and after the environmental tests.

\section{3 | DISCUSSION}

Table 1 compares the proposed QHA and previously reported QHAs: although a few reported QHAs achieve high 
performance, they are of large sizes. ${ }^{12,16-18}$ Certain reported QHAs are compact, yet their frequency ratios and sizes cannot satisfy the required parameters. ${ }^{10,14,15,19}$ Loading IUSHSs and the cross-shape strip enables the proposed antenna to achieve high broadside gain, small AR, and compact electrical size with a smaller frequency ratio. Furthermore, it provides costeffective solutions to spaceborne antennas by avoiding dependence on space-specific dielectric materials to realize miniaturization. Consequently, the proposed antenna is a good candidate for spaceborne DCS applications.

\section{4 | CONCLUSION}

A novel spaceborne miniaturized CP UHF dual-band QHA with a small frequency ratio was presented. A prototype working at $402 / 505 \mathrm{MHz}$ was designed, fabricated, measured, and in-orbit tested with a weight of $651 \mathrm{~g}$ and an effective size of $0.161 \times 0.161 \times 0.214 \lambda^{3}{ }_{402 \mathrm{MHz}}$, with a 1.24 frequency ratio. The measured gain and axial ratio of the proposed QHA were better than $5.32 \mathrm{dBi}$ and $2.18 \mathrm{~dB}$ at the operating band. The test results proved that the methods used to design the proposed antenna are effective.

\section{ACKNOWLEDGMENTS}

We thank Dr. Lijun Xue, Dr. Bibo Guo, and Dr. Shilong Wei at Shenzhen Aerospace Dongfanghong Satellite Ltd. for helpful discussions and suggestions.

\section{DATA AVAILABILITY STATEMENT}

Data openly available in a public repository that issues datasets with DOIs

\section{ORCID}

Kai Хиe (1D https://orcid.org/0000-0003-0745-2491

Shaowei Liao (1D https://orcid.org/0000-0003-0792-2000

Ruilong Zhu @ https://orcid.org/0000-0002-7700-4726

Quan Xue (D https://orcid.org/0000-0002-4226-2127

Liqin Ding (10) https://orcid.org/0000-0002-5181-2493

Yang Wang (1) https://orcid.org/0000-0001-9894-7478

Qing Guo (1D https://orcid.org/0000-0003-4209-5469

\section{REFERENCES}

[1] Antennas for satellite communications. Space Antenna Handbook; New Jersey, USA: John Wiley \& Sons, Inc.; 2012:466-510. https://doi.org/10.1002/9781119945147.ch12.

[2] Liao S, Xue Q. Miniaturized VHF/UHF dual-band circularly polarized four-element sequential-rotation array antenna based on alternately overlapped bent radiation-coupled dual-L antenna elements. IEEE Trans Antennas Propag. 2018;66(9):4924-4929.
[3] Small circularly polarized antenna. Circularly Polarized Antennas; New Jersey, USA: John Wiley \& Sons, Inc.; 2014:29-72. https://doi.org/10.1002/9781118790526.ch2.

[4] Multi-band circularly polarized antennas. Circularly Polarized Antennas; New Jersey, USA: John Wiley \& Sons, Inc.; 2014:131190. https://doi.org/10.1002/9781118790526.ch4.

[5] Tawk Y, Chahoud M, Fadous M, Costantine J, Christodoulou CG. The miniaturization of a partially 3-D printed quadrifilar helix antenna. IEEE Trans Antennas Propag. 2017;65(10):5043-5051.

[6] Amin M, Cahill R. Effect of helix turn angle on the performance of a half wavelength quadrifilar antenna. IEEE Microwave Wireless Compon Lett. 2006;16(6):384-386.

[7] Chew DKC, Saunders SR. Meander line technique for size reduction of quadrifilar helix antenna. IEEE Antennas Wireless Propag Lett. 2002;1:109-111.

[8] Zheng L, Gao S. Compact dual-band printed square quadrifilar helix antenna for global navigation satellite system receivers. Microwave Opt Technol Lett. 2011;53(5):993-997.

[9] Qin W, Shi L, Sun W, Yang W, Ge L, Chen J. A wideband LTCC quad-phase power dividing network and its application to ceramic-based quadrifilar helix antennas. IEEE Access. 2019;7: 141094-141103.

[10] Rabemanantsoa J, Sharaiha A. Size reduced multi-band printed quadrifilar helical antenna. IEEE Trans Antennas Propag. 2011; 59(9):3138-3143.

[11] Khajepour S, Ghaffarian MS, Moradi G. Design of novel multiband folded printed quadrifilar helical antenna for GPS/WLAN applications. Electron Lett. 2017;53(2):58-60.

[12] Letestu Y, Sharaiha A. Broadband folded printed quadrifilar helical antenna. IEEE Trans Antennas Propag. 2006;54(5):1600-1604.

[13] U.S. Patent 6421028. July 16, 2002. Dual frequency quadrifilar helix antenna, Ohgren Mikael, Inventor. SAAB Ericsson Space AB, assignee.

[14] Byun G, Choo H, Kim S. Design of a dual-band quadrifilar helix antenna using stepped-width arms. IEEE Trans Antennas Propag. 2015;63(4):1858-1862

[15] Yang Y, Guo J, Sun B, Huang Y. Dual-band slot helix antenna for global positioning satellite applications. IEEE Trans Antennas Propag. 2016;64(12):5146-5152.

[16] Hosseini M, Hakkak M, Rezaei P. Design of a dual-band quadrifilar helix antenna. IEEE Antennas Wireless Propag Lett. 2005;4:39-42.

[17] Han Y, Wang H, Wang Z, et al. Dual-band spiral printed quadrifilar helical antenna miniaturized by surface and inner dielectric loading. IEEE Access. 2019;7:30244-30251.

[18] Letestu Y, Sharaiha A. Multiband printed quadrifilar helical antenna. Electron Lett. 2010;46(13):885-886.

[19] Liu H, Shi M, Fang S, Wang Z. Design of low-profile dual-band printed quadrifilar helix antenna with wide beamwidth for UAV GPS applications. IEEE Access. 2020;8:157541-157548.

How to cite this article: Xue $\mathrm{K}$, Liao $\mathrm{S}$, Zhu R, et al. Spaceborne miniaturized UHF dual band helix antenna with a small frequency ratio. Microw Opt Technol Lett. 2021;1-7. https://doi.org/10.1002/mop. 32820 